\title{
Control Dependence for Extended Finite State Machines
}

\author{
Kelly Androutsopoulos ${ }^{1}$, David Clark ${ }^{1}$, Mark Harman ${ }^{1}$, Zheng Li $^{1}$, \\ and Laurence Tratt ${ }^{2}$ \\ ${ }^{1}$ Department of Computer Science, King's College London, Strand, London, \\ WC2R 2LS, United Kingdom \\ \{kalliopi.androutsopoulos, david.j.clark, mark.harman, zheng.li\}@kcl.ac.uk \\ ${ }^{2}$ Bournemouth University, Poole, Dorset, BH12 5BB, United Kingdom \\ laurie@tratt.net
}

\begin{abstract}
Though there has been nearly three decades of work on program slicing, there has been comparatively little work on slicing for state machines. One of the primary challenges that currently presents a barrier to wider application of state machine slicing is the problem of determining control dependence. We survey existing related definitions, introducing a new definition that subsumes one and extends another. We illustrate that by using this new definition our slices respect Weiser slicing's termination behaviour. We prove results that clarify the relationships between our definition and older ones, following this up with examples to motivate the need for these differences.
\end{abstract}

Keywords: extended finite state machines, reactive systems, control dependence, slicing.

\section{Introduction}

Program slicing is a source code analysis technique that identifies the parts of a program's source code which can affect the computation of a chosen variable at a chosen point in it. The variable and point of interest constitute the slicing criterion. There are many variations on the slicing theme. For instance, slices can be constructed statically (with respect to all possible inputs), dynamically (with respect to a single input) or within some spectrum in-between. Program slicing has proved to be widely applicable, with application areas ranging from program comprehension [HBD03] to reverse engineering and reuse [CCD98.

However, despite thirty years of research, several hundred papers and many surveys on program slicing [BH04, De 01, Tip95, there has been comparatively little work on slicing at the model level. This paper tackles slicing at the model level, particularly static slicing of Finite State Machines (FSMs).

FSMs are a graphical formalism that have become widely used in specifications of embedded and reactive systems. Their main drawback is that even moderately complicated systems result in large and unwieldy diagrams. Harel's Statecharts Har87 and Extended Finite State Machines (EFSMs) are two of the many attempts over past decades to address FSM's disadvantages. 
Work on slicing FSM models began with the work of Heimdahl et al. in the late 1990s [HW97, HTW98, followed by Wang et al. WDQ02 and then in 2003 by the work of Korel et al. KSTV03 and more recently by Langenhove and Hoogewijs [LH07] and by Labbé et al. LGP07, LG08].

One of the challenges facing any attempt to slice an EFSM is the problem of how to correctly account for control dependence. It is common for state machines modelling such things as reactive systems not to have a final computation point or 'exit node'. To overcome this problem, Ranganath et al. $\mathrm{RAB}^{+} 05$ recently introduced the concept of a control sink and associated control dependence definitions for reactive programs. A control sink is a strongly connected component from which control flow cannot escape once it enters. Building on this, Labbé et al. [LGP07, LG08 introduced a notion of control dependence and an associated slicing algorithm for EFSMs that was non-termination sensitive. However, they introduce a syntax dependent condition and thus cannot be applied to any FSM.

However, traditional control dependence, as used in program slicing HRB90. is non-termination insensitive, with the consequence that the semantics of a program slice dominates the semantics of the program from which it is; slicing may remove non-termination, but it will never introduce it. In moving slicing from the program level to the state based model level, an important choice needs to be made

\section{"should EFSM slicing be non-termination sensitive or insensitive?"}

Recent work on control dependence has only considered the non-termination sensitive option [LGP07, LG08]. The non-termination insensitive option was explored by Korel et al. KSTV03, but only for the restricted class of state machines that guarantee to have an exit state. Heimdahl et al. HW97, HTW98 have a different notion of control dependence which is not a structural property of the graph of FSMs but is based on the dependency relation between events and generated events. This could lead to slices being either non-termination sensitive or insensitive depending on the specification. The definition of control dependence given in WDQ02, LH07] is for UML statecharts with nested and concurrent states and is the same as that of data dependence when applied to EFSMs that do not have concurrent and/or nested states. This leaves open the question of how to extend control dependence to create non-termination insensitive slicing for general EFSMs in which there may be no exit node.

This problem is not merely of intellectual curiosity as it also has implications for the applications of slicing. In the literature on traditional program slicing, a non-termination sensitive formulation was proposed as early as 1993 by Kamkar Kam93, but has not been taken up in subsequent slicing research. Non-termination sensitive slicing tends to produce very large slices, because all iterative constructs that cannot be statically determined to terminate must be retained in the slice, no matter whether they have any effect other than termination on the values computed at the slicing criterion. These 'loop shells' must be retained in order to respect the definition of non-termination sensitivity. Furthermore, for most of the applications of slicing listed above, it turns out that it is perfectly acceptable for slicing to be non-termination insensitive. 
In this paper, we introduce a non-termination insensitive form of control dependence for EFSM dependence analysis, that can be applied to any FSM, and a slicing algorithm based upon it. Like Labbé et al., we build on the recent work of Ranganath et al. $\mathrm{RAB}^{+} 05$, but our definition is non-termination insensitive. Also, unlike Korel's definition, our development of the recent work of Ranganath et al. allows us to handle arbitrary EFSMs. We prove that our definition of control dependence is backward compatible with traditional non-termination insensitive control dependence outside of control sinks. Furthermore, we prove that our definition agrees with the non-termination sensitive control dependence of Labbé et al. inside control sinks. Finally we demonstrate the type of slices produced with our definition.

\section{Extended Finite State Machines}

We formally define an EFSM as follows.

Definition 1 (Extended Finite State Machine). An Extended Finite State Machine (EFSM) $E=(S, T, E v, V)$ where $S$ is a set of states, $T$ is a set of transitions, Ev is a set of events, and $V$ is a store represented by a set of variables. Transitions have a source state source $(t) \in S$, a target state target $(t) \in S$ and a label $l b l(t)$. Transition labels are of the form $e_{1}[g] /$ a where $e_{1} \in E v, g$ is a guard, i.e. a condition (we assume a standard conditional language) that guards the transition from being taken when an $e_{1}$ is true, and a is a sequence of actions (we assume a standard expression language including assignments). All parts of a label are optional.

EFSMs are possibly non-deterministic. States of $S$ are atomic. Actions can involve store updates or generation of events or both. A transition $t$ may have a successor $t^{\prime}$ whose source is the same as the target of $t$. Two or more distinct transitions which share the same source node are said to be siblings. A final transition is a transition whose target is an exit state and an exit state is a state which has no outgoing transitions. An $\varepsilon$-transition is one with no event or guard.

\section{$3 \quad$ Survey}

In this section we survey several existing definitions of control dependence and discuss their strengths and weaknesses.

Ranganath et al.'s control dependence definitions $\left.\mathrm{RAB}^{+} 05, \mathrm{RAB}^{+} 07\right]$ are defined for programs of systems with multiple exit points and / or which execute indefinitely, and therefore form the basis for subsequent state machine control dependence definitions. We exclude from this discussion the control dependence definition as given in WDQ02, LH07 because it is defined in terms of concurrent states and transitions and EFSMs do not have concurrent states and transitions. Moreover, when applied to states and transitions that are not concurrent, it is the same as data dependence as in Definition 13 . 
Korel et al [KSTV03, Ranganath et al. and Labbé et al. [LGP07, LG08] definitions of control dependence are given in terms of execution paths. Since a path is commonly presented as a (possibly infinite) sequence of nodes, a node is in a path if it is in the sequence. A transition is in a path if its source state is in the path and its target state is both in the path and immediately follows its source state. A maximal path is any path that terminates in an end node or final transition, or is infinite.

\subsection{Control Flow for RSML}

Heimdahl et al. HW97, HTW98 present an approach for slicing specifications modelled in the Requirements State Machine Language (RSML) [LHHR94, a tabular notation that is based on hierarchical finite state machines. Transitions have events, guards and actions; events can generate events as actions, which are broadcast in the next step of execution. Heimdahl et al. were the first to present a control dependence-like definition for FSMs; it differs from the traditional notion as it defines control flow in terms of events rather than transitions.

Definition 2 (Control flow for RSML (CF) [HTW98]). Let $E$ be the set of all events and $T$ the set of all transitions. The relation $\operatorname{trigger}(T \rightarrow E)$ represents the trigger event of a transition. The relation action $\left(T \rightarrow E^{2}\right)$ represents the set of events that make up the action caused by executing a transition. follows $(T \rightarrow T)$ is defined as: $\left(t_{1}, t_{2}\right) \in$ follows iff trigger $\left(t_{1}\right) \in \operatorname{action}\left(t_{2}\right)$.

CF can be applied to non-terminating systems that have multiple exit nodes. However, it depends on transitions being triggered by events and being able to generate events as actions and therefore cannot be applied to any finite state machine, such as EFSMs that do not generate events.

\subsection{Control Dependence for EFSMs}

Korel et al. KSTV03] present a definition of control dependence for EFSMs in terms of post dominance that requires execution paths to lead to an exit state.

Definition 3 (Post Dominance [KSTV03]). Let $Y$ and $Z$ be two states and $T$ be an outgoing transition from $Y$.

- State $Z$ post-dominates state $Y$ iff $Z$ is in every path from $Y$ to an exit state.

- State $Z$ post-dominates transition $T$ iff $Z$ is on every path from $Y$ to the exit state though $T$. This can be rephrased as $Z$ post-dominates target $(T)$.

Definition 4 (Insensitive Control Dependence (ICD) [KSTV03]). Transition $T_{k}$ is control dependent on transition $T_{i}$ if:

1. $\operatorname{source}\left(T_{k}\right)$ post-dominates transition $T_{i}\left(\operatorname{or} \operatorname{target}\left(T_{i}\right)\right)$, and

2. $\operatorname{source}\left(T_{k}\right)$ does not post-dominate source $\left(T_{i}\right)$.

This definition is successful in capturing the traditional notion of control dependence for static backward slicing. However it can only determine control dependence for state machines with exactly one end state, failing if there are multiple exit states or if the state machine is possibly non-terminating. 


\subsection{Control Dependence for Non-terminating Programs}

Ranganath et al. $\mathrm{RAB}^{+} 05, \mathrm{RAB}^{+} 07$ address the issue of determining control dependence for programs utilising Control Flow Graphs (CFGs). A CFG is a labelled, directed graph with a set of nodes that represent statements in a program and edges that represent the control flow. A node is either a statement node (which has a single successor) or a predicate node (which has two successors, labelled with $T$ or $F$ for the true and false cases respectively). A CFG has a start node $n_{s}$ (which must have no incoming edges) such that all nodes are reachable from $n_{s}$; it may have a set of end nodes that have no successors.

Two versions of control dependence definitions are described: non-termination sensitive and non-termination insensitive control dependence. The difference between these definitions lies in the choice of paths. Non-termination sensitive control dependence is given in terms of maximal paths.

\section{Definition 5 (Non-termination Sensitive Control Dependence}

(NTSCD)). In a CFG, $N_{i} \stackrel{N T S C D}{\longrightarrow} N_{j}$ means that a node $N_{j}$ is non-termination sensitive control dependent on a node $N_{i}$ iff $N_{i}$ has at least two successors $N_{k}$ and $N_{l}$ such that: for all maximal paths $\pi$ from $N_{k}$, where $N_{j} \in \pi$; and there exists a maximal path $\pi_{0}$ from $N_{l}$ where $N_{j} \notin \pi_{0}$.

Non-termination insensitive control dependence is given in terms of sink-bounded paths that end in control sinks. A control sink is a region of the graph which, once entered, is never left. These regions are always SCCs, even if only the trivial SCC, i.e. a single node with no successors.

Definition 6 (Control Sink). A control sink, $\mathcal{K}$, is a set of nodes that form a strongly connected component such that, for each node $n$ in $\mathcal{K}$ each successor of $n$ is in $\mathcal{K}$.

Definition 7 (Sink-bounded Paths). A maximal path $\pi$ is sink-bounded iff there exists a control sink $\mathcal{K}$ such that:

1. $\pi$ contains a node from $\mathcal{K}$;

2. if $\pi$ is infinite then all nodes in $\mathcal{K}$ occur infinitely often.

The second clause of Definition 7 defines a form of fairness and hence we refer to it as the fairness condition. $\operatorname{SinkPaths}(N)$ denotes a set of sink-bounded paths from a node $N$. We now define Ranganath et al. $\left[\mathrm{RAB}^{+} 05\right]$ non-termination insensitive version of control dependence.

\section{Definition 8 (Non-termination Insensitive Control Dependence}

(NTICD)). In a CFG, $T_{i} \stackrel{\text { NTICD }}{\longrightarrow} N_{j}$ means that a node $N_{j}$ is non-termination insensitive control dependent on a node $N_{i}$ iff $N_{i}$ has at least two successors $N_{k}$ and $N_{l}$ such that:

1. for all paths $\pi \in \operatorname{SinkPaths}\left(N_{k}\right)$ where $N_{j} \in \pi$;

2. there exists a path $\pi_{0} \in \operatorname{SinkPaths}\left(N_{l}\right)$ where $N_{j} \notin \pi_{0}$. 


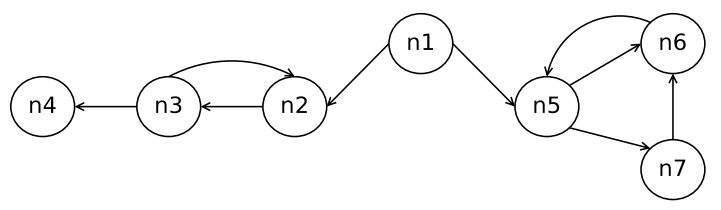

Fig. 1. A CFG with multiple exit points and which is potentially non-terminating

The difference between paths in NTSCD and NTICD is shown in Figure 1 . According to Definition [5, $n 1 \stackrel{\text { NTSCD }}{\longrightarrow} n 2$ and $n 1 \stackrel{\text { NTSCD }}{\longrightarrow} n 3$ but not $n 1 \stackrel{\text { NTSCD }}{\longrightarrow} n 4$ because $n 4$ is not in all maximal paths as there is a maximal path with an infinite loop, i.e. $\{n 2 \rightarrow n 3 \rightarrow n 2 \ldots\}$. However, $n 1 \stackrel{\text { NTICD }}{\longrightarrow} n 2, n 3, n 4$ since $n 2, n 3$ and $n 4$ occur on all sink-bounded paths from $n 2$ (the control sink for these paths is $n 4$ ) and there exists a sink bounded path from $n 5$ (the control sink consists of $n 5, n 6, n 7)$ which does not include $n 2, n 3$ and $n 4$. Compared to NTSCD, NTICD cannot calculate any control dependencies within control sinks. For example, in Figure 1, $n 5 \stackrel{\text { NTSCD }}{\longrightarrow} n 7$ but no such dependency exists for NTICD. Some programs (e.g. servers) are a global control sink and as such there would be NTSCD, but no NTICD, dependences.

\subsection{Control Dependence for Communicating Automata}

Labbé et al. LG08] adapt Ranganath et al.'s NTSCD definition for communicating automata, in particular focusing on Input/Output Symbolic Transition Systems (IOSTS) GGRT06.

Definition 9 (Labbé et al.- Non-Termination Sensitive Control Dependence (LG-NTSCD) [LG08]). For an IOSTS $S$, a transition $T_{j}$ is control dependent on a transition $T_{i}$ if $T_{i}$ has a sibling transition $T_{k}$ such that:

1. $T_{i}$ has a non-trivial guard, i.e. a guard whose value is not constant under all variable valuations;

2. for all maximal paths $\pi$ from $T_{i}$, the source of $T_{j}$ belongs to $\pi$;

3. there exists a maximal path $\pi_{0}$ from $T_{k}$ such that the source of $T_{j}$ does not belong to $\pi_{0}$.

FSM models differ from CFGs is several ways. For example, FSMs can have multiple start and exit nodes, more than two edges between two states and more than two successors from a state. Moreover, in CFGs, decisions (Boolean conditions) are made at the predicate nodes while in state machines they are made on transitions. Labbé et al. take such differences into account when adapting NTSCD. For example in Figure $2 T 2 \stackrel{\text { NTSCD }}{\longrightarrow} T 3$ and $T 3 \stackrel{\text { NTSCD }}{\longrightarrow} T 2$ because according to the second clause in Definition 5 the maximal paths start from start. However these control dependencies are non-sensical because T2 and T3 are

${ }^{1}$ Labbé et al.'s definition of control dependence in [LGP07] differs slightly from Labbé et al. LG08, so we evaluate the most recent. 


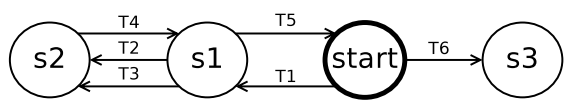

Fig. 2. If NTSCD or NTICD is applied, undesired dependences are produced

sibling transitions. Using LG-NTSCD these control dependencies do not exist because in the third clause of Definition 9 the maximal paths start from $s 1$.

The first clause of LG-NTSCD concerning the non-triviality of guards is introduced in order to avoid a transition being control dependent on transitions that are executed non-deterministically even though they are NTSCD control dependent. Furthermore, because this is a syntax dependent clause, the definition cannot be applied to many FSMs, such as the FSM for the elevator system in Figure 3 that contains transitions with trivial guards.

\section{New Control Dependence Definition: UNTICD}

We define a new control dependence definition by extending Ranganath et al.'s NTICD definition and subsuming Korel et al.'s definition in order to capture a notion of control dependence for EFSMs that has the following properties. First, the definition is general in that it should be applicable to any reasonable FSM language variant. Second, it is applicable to non-terminating FSMs and / or those that have multiple exit states. Third, by choosing FSM slicing to be nontermination insensitive (in order to coincide with traditional program slicing) it produces smaller slices than traditional non-termination sensitive slicing.

Following $\left[\mathrm{RAB}^{+} 05\right.$, the paths that we consider are sink-bounded paths, i.e. those that terminate in a control sink as in Definition 6. Unlike NTICD, the sinkbounded paths are unfair, i.e. we drop the fairness condition in Definition 7 , For non-terminating systems this means that control dependence can be calculated within control sinks.

Definition 10 (Unfair Sink-bounded Paths). A maximal path $\pi$ is sinkbounded iff there exists a control sink $\mathcal{K}$ such that $\pi$ contains a transition from $\mathcal{K}$.

Note that a transition is in a path if its source state is in the path and its target state is both in the path and immediately follows its source state.

\section{Definition 11 (Unfair Non-termination Insensitive Control Depend-} ence (UNTICD)). $T_{i} \stackrel{U N T I C D}{\longrightarrow} T_{j}$ means that a transition $T_{j}$ is control dependent on a transition $T_{i}$ iff $T_{i}$ has at least one sibling $T_{k}$ such that:

1. for all paths $\pi \in$ UnfairSinkPaths(target $\left.\left(T_{i}\right)\right)$, the source $\left(T_{j}\right)$ belongs to $\pi$;

2. there exists a path $\pi \in$ UnfairSinkPaths $\left(\operatorname{source}\left(T_{k}\right)\right)$ such that the source $\left(T_{j}\right)$ does not belong to $\pi$. 
UNTICD is in essence a version of NTICD modified to EFSMs (rather than CFGs) and given in terms of unfair sink-bounded paths. This means that, unlike in the second clause of Definition 8, sink-bounded paths start from the source of $T_{k}$ rather than from the target of $T_{k}$ because EFSMS can have many transitions between states and Definition 8 would lead to non-sensical dependences, e.g. in Figure $2 T 2 \stackrel{\text { NTICD }}{\longrightarrow} T 3$ while according to our definition $T 2$ does not control $T 3$.

\section{Properties of the Control Dependence Relation}

We prove the following properties for UNTICD: UNTICD subsumes ICD; the transitive closure for the NTICD relation is contained in the transitive closure for the UNTICD relation; and for an EFSM $M$, UNTICD and NTSCD dependences for all transitions within control sinks are identical.

\subsection{UNTICD Subsumes ICD}

Proposition 1 Definition 4 (ICD) is a special case of Definition 11 (UNTICD).

Proof. Definition 4 is given in terms of post dominance which considers every path to a unique exit state. Definition 11 is given in terms of sink-bounded paths that terminate in control sinks. The final transition that leads to the exit state is a trivial strongly-connected component that has no successors, and hence is a control sink. Therefore, the paths in ICD are contained in the paths of NTICD, but NTICD is not restricted to these. Moreover, the clauses of definition 4 are the same as the clauses of definition 11 .

\subsection{Relation between NTICD and UNTICD's Transitive Closures}

In Theorem 2 we show that the transitive closure of $\stackrel{\text { NTICD }}{\longrightarrow}$ is contained in the transitive closure of $\stackrel{\mathrm{UNTICD}}{\longrightarrow}$. This shows that UNTICD does not introduce any additional dependences other than NTICD outside of the control sinks (see Lemma 2) but introduces dependences within control sinks. In order to prove this theorem, we first need to identify the regions in the state machine where dependencies can occur and we do that by considering all the cases in which a transition $t 1$ controls another transition $t 2$, where $K, K 1, K 2$ are control sinks:

$$
\begin{array}{r}
\forall K . t 1 \notin K \wedge t 2 \notin K \\
\exists K . t 1 \in K \wedge t 2 \in K \\
\exists K 1, K 2 . t 1 \in K 1 \wedge t 2 \in K 2 \\
\forall K . t 1 \notin K \wedge \exists K . t 2 \in K \\
\forall K . t 2 \notin K \wedge \exists K . t 1 \in K
\end{array}
$$

In case (1) both $t 1$ and $t 2$ are not in any control sink $K$. In case (2) both $t 1$ and $t 2$ are in the same control sink $K$. In case (3) $t 1$ is in a control sink $K 1$ and $t 2$ is in another control sink $K 2$. In case (4) $t 1$ is not in any control sink and $t 2$ 
belongs to a control sink. In case (5) $t 2$ does not belong to any control sink and $t 1$ belongs to a control sink. We introduce Definition 12 that defines a descendant of a transition and the Lemma 1 so that we can discard any impossible cases.

Definition 12 (Descendent). A descendant of $t$ is a transition related to $t$ by the closure of the successor relation.

Lemma 1. For all transitions $t$ in a control sink $K$, all descendants of $t$ belong to $K$.

Proof. By Definition [6 of the control sink and Definition 12 of the descendant relation.

By Lemma 1) cases (3) and (5) are not possible since $t 1$ can only control $t 2$ if $t 2$ is a descendant of $t 1$. Therefore, we only consider cases (11), (2), and (4). When $t 1$ NTICD controls $t 2$, then for each case we write case $\mathbb{1}^{F}$, case $2^{F}$, and case $44^{F}$.

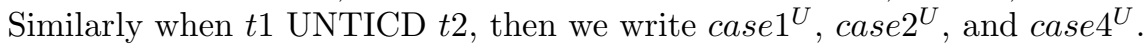

Lemma 2 shows that the control dependences produced by applying UNTICD to transitions outside of the control sink are the same as those produced when applying NTICD, i.e. cas $\mathbb{1}^{H}=\operatorname{cas} \in \mathbb{1}^{F}$.

Lemma 2. For an EFSM M, NTICD and UNTICD dependences for transitions $T$ outside of the control sink $K$ (where $t \in T$ and $t \notin K$ ), are the same.

Proof. Let us assume that in an EFSM $M, T_{j}$ is NTICD control dependent on $T_{i}\left(T_{i} \stackrel{N T I C D}{\longrightarrow} T_{j}\right)$ and that $T_{i}$ and $T_{j}$ are outside of the control sink. From Definition 8 , $T_{i}$ has a sibling transition $T_{k}$ such that there exists a path $\pi_{k} \in$ $\operatorname{SinkPaths}\left(T_{k}\right)$ where the $\operatorname{source}\left(T_{j}\right)$ does not belong to $\pi_{k}$.

Now suppose that the fairness condition in the definition of sink bounded paths is removed, i.e. Definition 11 holds, then this affects the transitions within the control sink only in that they do not occur infinitely often. The source of $T_{j}$ still remains on all paths from $T_{i}$ as these are outside of the control sink and $\pi_{k}$ still exists. Therefore, NTICD and UNTICD dependences of transitions outside of the control sink are the same.

The pairwise intersection of case (11), (2), (4) are empty. Therefore the relations can be partitioned as follows:

$$
\begin{aligned}
& \text { case } \mathbb{1}^{F} \cup \operatorname{cas} 2^{F} \cup \operatorname{cas} \notin 4^{F}=\stackrel{\text { NTICD }}{\longrightarrow}
\end{aligned}
$$

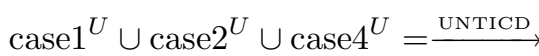

In Theorem 2 we show that the transitive closure of NTICD dependences between transitions within a control sink and between a transition outside of the control sink and a transition within a control sink is a subset of the transitive closure of UNTICD dependences between transitions within a control sink and between a transition outside of the control sink and a transition within a control sink, i.e. $\left(\operatorname{cas} 2^{F} \cup \operatorname{cas} 44^{F}\right)^{*} \subseteq\left(\operatorname{cas} 2^{U} \cup \text { cas } 4{ }^{H}\right)^{*}$. First we prove the following lemma.

Lemma 3. Let $A \cap B=C \cap D=\emptyset$ and $X=A \cup B$ while $Y=C \cup D$. If $A=C$ then $X^{*} \subseteq Y^{*}$ if $B^{*} \subseteq D^{*}$. All relations are over the same base set. 
Proof. $\left(x_{1}, x_{2}\right) \in X^{*}$ iff there exists a path $\pi \in\left(x_{1}, x_{2}\right),\left(x_{2}, x_{3}\right), \ldots,\left(x_{n-1}, x_{n}\right)$ so that for two successive members $\left(x_{i}, x_{j}\right)$ and $\left(x_{k}, x_{l}\right) \in \pi, x_{j}=x_{k}$, and for all $\left(x_{i}, x_{j}\right) \in X$. This constructs the smallest transitive closure of $X$.

We show $X^{*} \subseteq Y^{*}$ by induction on the length of the path $\pi$ in $X^{*}$.

Base Case: length $(\pi)=1$ then either $\left(x_{0}, x_{1}\right) \in A=C \subseteq(C \cup D)^{*}=Y^{*}$ or $\left(x_{0}, x_{1}\right) \in X^{*}$ because $\left(x_{0}, x_{1}\right) \in B \subseteq B^{*} \subseteq D^{*} \subseteq(C \cup D)^{*}=Y^{*}$

Induction Case: (Inductive Hypothesis (IH)) Let $x X^{*} y$ because there exists a path $\pi \in x X^{*} x_{1} X^{*} x_{2} \ldots X^{*} y$ of length $N$ in $X^{*}$. Then there exists a path $\pi_{1}$ in $Y$ such that $x Y^{*} y$.

Let $x X^{*} z$ because there exists a path $\pi$ of length $N+1$ in $X$. Then $\exists y, z$. $x X^{*} y X z$ and by IH $x Y^{*} y$ by the same arguments for the base case of $y X z$ then $y Y^{*} z$ hence $x Y^{*} z$.

Theorem 2. The transitive closure of NTICD, is contained in the transitive closure of UNTICD. $\stackrel{\text { NTICD }}{\longrightarrow} \subseteq \stackrel{U N T I C D}{\longrightarrow}^{*}$

Proof. $\stackrel{\text { NTICD }}{\longrightarrow} \subseteq \stackrel{\text { UNTICD }}{\longrightarrow}^{*}$ can also be expressed as the transitive closure for all of the cases: $\left(\operatorname{case} 1^{F} \cup \text { case } 2^{F} \cup \text { case } 4^{F}\right)^{*} \subseteq\left(\operatorname{case} \mathbb{1}^{\cup} \cup \text { case }{ }^{U} \cup \text { case } 4\right)^{*}$ which is true if:

- case $\mathbb{1}^{F}=$ case 1 , i.e. that NTICD and UNTICD dependences between transitions that are not in a control sink are the same, by Lemma 2 , and

- $\left(\operatorname{case} 2^{F} \cup \operatorname{cas} 4^{F}\right)^{*} \subseteq\left(\operatorname{case} 2^{U} \cup \text { case } 4\right)^{*}$, i.e. that the transitive closure of NTICD dependences between transitions within a control sink and a transition outside of the control sink, and between transitions within a control sink is a subset of the transitive closure of UNTICD dependences between transitions within a control sink and a transition outside of the control sink, and between transitions within a control sink. This is true because of Lemma 3

\subsection{NTSCD and UNTICD Dependencies within Control Sinks}

Finally, we show that UNTICD and NTSCD are compatible in control sinks.

Theorem 3. For every $T_{i} \in K$ and $T_{j} \in K$ where $K$ is a control sink in EFSM $M, T_{i} \stackrel{U N T I C D}{\longrightarrow} T_{j}$ iff $T_{i} \stackrel{N T S C D}{\longrightarrow} T_{j}$.

Proof. In a control sink $K$, if $T_{i} \in K$ and $T_{j} \in K$, then according to Definition[11 sink-bounded paths are reduced to maximal paths, since transitions in $K$ do not occur infinitely often (fairly). This coincides with Definition 5. Therefore, the control dependences produced by UNTICD and NTSCD for transitions within control sinks are equivalent.

\section{Comparison of UNTICD with Existing Definitions}

Figure 3 illustrates an EFSM of the door control component, a subcomponent of the elevator control system SW99]. The door component controls the elevator 


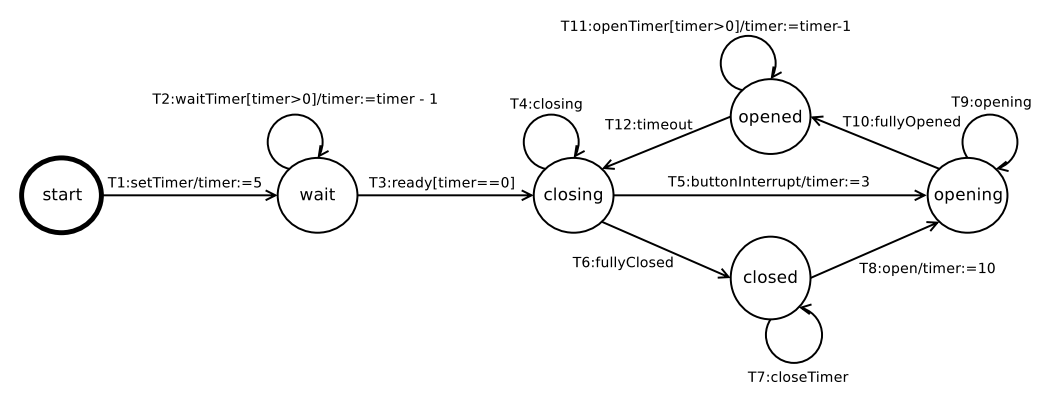

Fig. 3. An EFSM specification for the door control of the elevator system

\begin{tabular}{l|lr} 
CF & No dependences as the EFSM does not have generated events \\
\hline ICD & Not applicable as the EFSM does not have a unique exit state \\
\hline NTSCD & $\begin{array}{l}\text { wait } \rightarrow \text { closing } \\
\text { closed } \rightarrow \text { opening } \\
\text { opened } \rightarrow \text { closing }\end{array}$ & $\begin{array}{r}\text { closing } \rightarrow \text { closed } \\
\text { opening } \rightarrow \text { opened } \\
\text { closing } \rightarrow \text { opening }\end{array}$ \\
\hline NTICD & No dependences & \\
\hline LG-NTSCD & $T 3 \rightarrow T 4, T 5, T 6$ & $T 6 \rightarrow T 7, T 8$ \\
UNTICD & $T 5 \rightarrow T 9, T 10$ & $T 10 \rightarrow T 11, T 12$ \\
& $T 8 \rightarrow T 9, T 10$ & \\
& $T 12 \rightarrow T 4, T 5, T 6$ &
\end{tabular}

Fig. 4. Control dependences computed by new and existing definitions for Figure 3

door, i.e. it opens the door, waits for the passengers to enter or leave the elevator and finally shuts the door. In this section we compute all the control dependencies for this EFSM using the existing and new definitions for the purpose of comparison, as given in Figure 4 .

CF cannot be applied to the EFSM in Figure 3 because it is given in terms of the relationship between events and generated events and according to the syntax of EFSMs, events cannot be generated.

ICD cannot be applied to the the EFSM in Figure 3 because it does not have a unique exit state. For EFSMs that lead to a unique exit state the control dependences computed for both ICD and UNTICD are the same. For example, in Figure 2, ICD and UNTICD compute the same dependences, i.e. $T 1 \rightarrow T 2, T 3, T 4, T 5$.

In Figure 4. NTSCD and NTICD are given in terms of nodes but can easily be represented in terms of transitions. Compared to UNTICD, NTSCD considers maximal paths rather than sink-bounded paths and consequently introduces more dependences when there are loops on paths that lead to a control sink. For example, in Figure 3, wait $\stackrel{\text { NTSCD }}{\longrightarrow}$ closing because of the loop introduced by the self-transition T2. Note that NTSCD and UNTICD have the same dependences inside control sinks - we have formally shown this to be true in Theorem 3 .

In Figure 3 there are no NTICD dependences because any control dependency caused by loops on paths to a control sink are ignored and there are 
no control dependencies within control sinks because of the fairness condition of sink-bounded paths. Unlike NTICD, UNTICD calculates dependences with control sinks. Also, as formally shown by Theorem 2 the transitive closure of NTICD is contained within the transitive closure of UNTICD, although trivially true in this case.

LG-NTSCD is NTSCD adapted for transitions and with a syntax dependent clause, i.e. that the controlling transition's guard must be non-trivial. This additional clause reduces the number of dependences compared to those of NTSCD. For example, in Figure 3, T5, T6, T8, T10 and T12 do not control any other transition because they have trivial guards. The transitive closure of LG-NTSCD as for slicing, could produce too few results to be useful.

\section{$7 \quad$ EFSM Slicing with UNTICD}

Backward static program slicing was first introduced by Weiser Wei81 and describes a source code analysis technique that, through dependence relations, identifies all the statements in the program that influence the computation of a chosen variable and point in the program, i.e. the slicing criterion. It is nontermination insensitive. Similarly, EFSM slicing identifies those transitions which affect the slicing criterion, by computing control dependence and data dependence. Data dependence is a definition-clear path between a variable's definition and use. We adopt the data dependence definition of [KSTV03] for an EFSM.

Definition 13 (Data Dependence (DD)). $T_{i} \stackrel{D D}{\longrightarrow} T_{k}$ means that transitions $T_{i}$ and $T_{k}$ are data dependent with respect to variable $v$ if:

1. $v \in D\left(T_{i}\right)$, where $D\left(T_{i}\right)$ is a set of variables defined by transition $T_{i}$, i.e. variables defined by actions and variables defined by the event of $T_{i}$ that are not redefined in any action of $T_{i}$;

2. $v \in U\left(T_{k}\right)$, where $U\left(T_{i}\right)$ is a set of variables used in a condition and actions of transition $T_{i}$;

3. there exists a path in an EFSM from the source $\left(T_{i}\right)$ to the target $\left(T_{k}\right)$ whereby $v$ is not modified.

The data dependences for the door controller EFSM in Figure 3 are: $\{T 1 \rightarrow$ $T 2, T 3\},\{T 2 \rightarrow T 2, T 3\},\{T 5 \rightarrow T 11\},\{T 8 \rightarrow T 11\}$, and $\{T 11 \rightarrow T 11\}$.

Definition 14 (Slicing Criterion). A slicing criterion for an EFSM is a pair $(t, V)$ where transition $t \in T$ and variable set $V \subseteq V$ ar. It designates the point in the evaluation immediately after the execution of the action contained in transition $t$.

Definition 15 (Slice). A slice of an EFSM M, is an EFSM, $M^{\prime}$, that contains $\varepsilon-$ transitions. The transitions that are not $\varepsilon$-transitions are in the set of transitions that are directly or indirectly (transitive closure) DD and UNTICD on the slicing criterion $c$. 


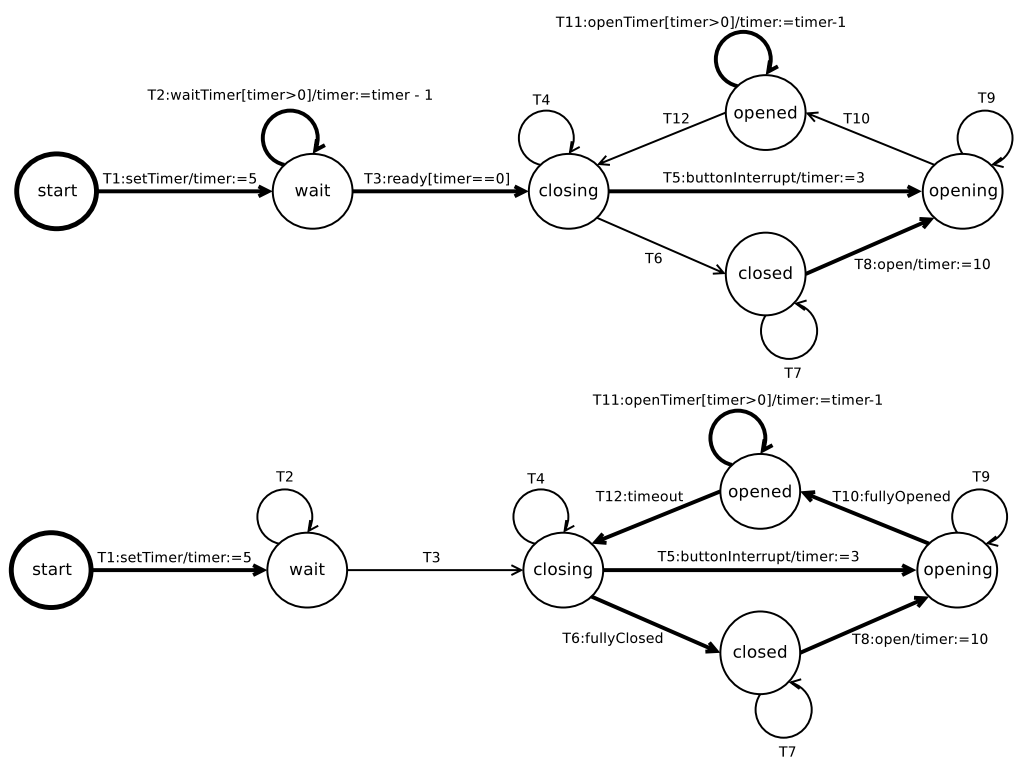

Fig. 5. Static slices computed with LG-NTSCD (top) and UNTICD (bottom). Marked transitions are in bold. LG-NTSCD has less marked transitions than UNTICD because dependences in the control sink are not valid as transitions have trivial guards.

\subsection{Computing EFSM Slices}

The objective of the slicing algorithm is to automatically compute the slice of an EFSM model $M$ with respect to the given slicing criterion $c$. First, the algorithm computes the data dependences, using Definition [13, and the control dependences, using Definition 11, for all transitions in $M$. These are then represented in a dependence graph, which is a directed graph where nodes represent transitions and edges represent data and control dependences between transitions. Then, given the slicing criterion $c$, the algorithm marks all backwardly reachable transitions from $c$, i.e. the transitive closure of DD and UNTICD with respect to $c$. All unmarked transitions are anonymised i.e. become $\varepsilon$-transitions. Note that we can replace UNTICD, with NTICD, LG-NTSCD and NTSCD in order to compare the different slices produced.

If the slicing criterion for the EFSM in Figure 3 is T11, then Figure 5(a) illustrates the slice produced when using UNTICD, and Figure 5(b) illustrates the slice produced when using LG-NTSCD. Unlike LG-NTSCD and NTSCD, UNTICD slicing slices away transitions which are affected by loops (before control sinks) that do not data dependent on T11, i.e. T3. Moreover, there are no LGNTSCD dependences within the control sink because the transitions have trivial guards. Trivial guards in Figure 3 do not affect whether $T 10$ and $T 9$ will be taken non-deterministically, so in the case where event opening occurs infinitely, T11 is never reached. If the slicing criterion for the EFSM in Figure 3 is T12, then the marked transitions in the UNTICD slice are $\{T 5, T 10, T 12\}$, while in 
the LG-NTSCD slice are $\{T 3, T 12\}$, in the NTSCD slice are $\{T 3, T 5, T 10, T 12\}$ and in the NTICD slice is $\{T 12\}$.

\section{Conclusions}

In this paper, we introduced a non-termination insensitive form of control dependence for EFSM slicing, that built on the recent work of Ranganath et al. $\mathrm{RAB}^{+} 05$ and subsumed Korel et al's definition [KSTV03. We demonstrated that by removing the fairness condition of Ranganath et al.'s NTICD no control dependences were removed, but extra control dependences within control sinks were introduced. Unlike NTICD our new definition works with non-terminating systems and, in general, produces smaller slices than those based on NTSCD.

\section{References}

[BH04] Binkley, D., Harman, M.: A survey of empirical results on program slicing. Advances in Computers 62, 105-178 (2004)

[CCD98] Canfora, G., Cimitile, A., De Lucia, A.: Conditioned program slicing. Information and Software Technology 40(11), 595-607 (1998)

[De 01] De Lucia, A.: Program slicing: Methods and applications. In: International Workshop on Source Code Analysis and Manipulation, pp. 142-149. IEEE Computer Society Press, Los Alamitos (2001)

[GGRT06] Gaston, C., Le Gall, P., Rapin, N., Touil, A.: Symbolic execution techniques for test purpose definition. In: Uyar, M.Ü., Duale, A.Y., Fecko, M.A. (eds.) TestCom 2006. LNCS, vol. 3964, pp. 1-18. Springer, Heidelberg (2006)

[Har87] Harel, D.: Statecharts: A visual formalism for complex systems. Science of Computer Programming 8(3), 231-274 (1987)

[HBD03] Harman, M., Binkley, D., Danicic, S.: Amorphous program slicing. Journal of Systems and Software 68(1), 45-64 (2003)

[HRB90] Horwitz, S., Reps, T., Binkley, D.: Interprocedural slicing using dependence graphs. ACM Transactions on Programming Languages and Systems 12(1), 26-61 (1990)

[HTW98] Heimdahl, M.P.E., Thompson, J.M., Whalen, M.W.: On the effectiveness of slicing hierarchical state machines: A case study. In: EUROMICRO 1998: Proceedings of the 24th Conference on EUROMICRO, p. 10435. IEEE Computer Society, Washington (1998)

[HW97] Heimdahl, M.P.E., Whalen, M.W.: Reduction and slicing of hierarchical state machines. In: Jazayeri, M. (ed.) ESEC 1997 and ESEC-FSE 1997. LNCS, vol. 1301, pp. 450-467. Springer, Heidelberg (1997)

[Kam93] Kamkar, M.: Interprocedural dynamic slicing with applications to debugging and testing. PhD Thesis, Department of Computer Science and Information Science, Linköping University, Sweden (1993)

[KSTV03] Korel, B., Singh, I., Tahat, L., Vaysburg, B.: Slicing of state-based models. In: Proceedings of the International Conference on Software Maintenance, pp. 34-43 (2003) 
[LG08] Labbé, S., Gallois, J.-P.: Slicing communicating automata specifications: polynomial algorithms for model reduction. Formal Aspects of Computing (2008)

[LGP07] Labbe, S., Gallois, J.-P., Pouzet, M.: Slicing communicating automata specifications for efficient model reduction. In: Proceedings of ASWEC, pp. 191-200. IEEE Computer Society, Los Alamitos (2007)

[LH07] Van Langenhove, S., Hoogewijs, A.: SV $\mathrm{L}_{t}$ : System verification through logic tool support for verifying sliced hierarchical statecharts. In: Fiadeiro, J.L., Schobbens, P.-Y. (eds.) WADT 2006. LNCS, vol. 4409, pp. 142-155. Springer, Heidelberg (2007)

[LHHR94] Leveson, N.G., Heimdahl, M.P.E., Hildreth, H., Reese, J.D.: Requirements Specification for Process-Control Systems. IEEE Transactions on Software Engineering 20(9), 684-706 (1994)

$\left[\mathrm{RAB}^{+} 05\right]$ Ranganath, V.P., Amtoft, T., Banerjee, A., Dwyer, M.B., Hatcliff, J.: A new foundation for control-dependence and slicing for modern program structures. In: Sagiv, M. (ed.) ESOP 2005. LNCS, vol. 3444, pp. 77-93. Springer, Heidelberg (2005)

$\left[\mathrm{RAB}^{+} 07\right]$ Ranganath, V.P., Amtoft, T., Banerjee, A., Hatcliff, J., Dwyer, M.B.: A new foundation for control dependence and slicing for modern program structures. ACM Trans. Program. Lang. Syst. 29(5), 27 (2007)

[SW99] Strobl, F., Wisspeintner, A.: Specification of an elevator control system an autofocus case study. Technical Report TUM-I9906, Technische Universität München (1999)

[Tip95] Tip, F.: A survey of program slicing techniques. Journal of Programming Languages 3(3), 121-189 (1995)

[WDQ02] Wang, J., Dong, W., Qi, Z.-C.: Slicing hierarchical automata for model checking UML statecharts. In: George, C.W., Miao, H. (eds.) ICFEM 2002. LNCS, vol. 2495, pp. 435-446. Springer, Heidelberg (2002)

[Wei81] Weiser, M.: Program slicing. In: $5^{\text {th }}$ International Conference on Software Engineering, pp. 439-449, San Diego, CA (March 1981) 